\title{
Lessons from the Contraceptive CHOICE Project: the Hull long-acting reversible contraception (LARC) initiative
}

\author{
James Trussell, ${ }^{1}$ Kate Guthrie ${ }^{2}$
}

\section{${ }^{1}$ Professor of Economics and Public Affairs and Faculty Associate, Office of Population Research, Princeton University, Princeton, NJ, USA \\ ${ }^{2}$ Consultant in Sexual and Reproductive Health, Sexual and Reproductive Healthcare Partnership, Hull, UK \\ Correspondence to Professor James Trussell, Office of Population Research, Princeton University, 202 Wallace Hall, Princeton University, Princeton, NJ 08544, USA; trussell@princeton.edu}

Received 27 March 2014 Revised 26 June 2014 Accepted 21 August 2014 Published Online First 18 September 2014
CrossMark

\footnotetext{
To cite: Trussell J, Guthrie K. I Fam Plann Reprod Health Care 2015;41:60-63.
}

\begin{abstract}
Aim To discover whether a hand-out explaining the benefits of intrauterine contraceptives (IUCS) and implants could increase their uptake in Hull, UK.

Methods We developed a simple double-sided A4 hand-out. On one side was a script with pictures of copper and levonorgestrel IUCs next to a 20 pence coin and of an implant beside a hairgrip. On the other side was the three-tiered effectiveness chart published in the textbook Contraceptive Technology. We implemented the

Key message points

- A very simple and cheap intervention can increase uptake of intrauterine contraceptives and implants.

- Management to ensure implementation and to monitor the intervention thereafter is crucial.

- Staff buy-in and ongoing engagement are essential to the success of the initiative.
\end{abstract} project in family planning (FP), abortion and antenatal clinics and general practitioner (GP) surgeries. The plan was that the receptionist would give the hand-out to every woman and ask her to read it before seeing a clinician. We evaluated the hand-out in FP clinics and GP practices because routine electronic monitoring reports were available only for these locations. Results There was no impact in GP practices. There was no overall impact in FP clinics, with the exception of the service hub, in which there was an increase in the proportion of women receiving IUCS or implants of $15.0 \%$ between the periods October 2011-April 2012 and May 2012-November 2012 ( $p=0.0002)$. This clinic is open 6 days per week and has permanent sexual health staff on the reception desk. The proportion of women receiving IUCS or implants returned to baseline in December 2012November 2013, when a change in clinic procedure to reduce waiting times caused staff to stop dispensing hand-outs.

Conclusions This was not a formal study, so there was no research coordinator to monitor the project. We think that there was no impact among GPs because the project was not implemented by them. The project was poorly implemented at the four satellite FP clinics. Only the service hub implemented the project, where it had a clear impact. We conclude that when implemented as intended, this simple, very low-cost long-acting reversible contraception intervention was highly effective and also extremely cost effective.

\section{INTRODUCTION}

Increasing the use of intrauterine contraceptives (IUCs) and implants is a national goal in the UK as well as a local goal in the city of Hull, because they are the most effective, and cost-effective, reversible contraceptive methods. Increased use of such methods would reduce the rate of unintended pregnancy. ${ }^{12}$ Our initiative was modelled on the Contraceptive CHOICE Project in St Louis, MO, USA. Participants in St Louis who wanted to commence contraception or to change contraceptive method were offered free contraception and read a brief introductory script when inquiring about the project and when enrolling. The goal was to increase use of IUCs to $6-10 \%$ and implants to $3 \%$ or more. ${ }^{3}$ The results dramatically exceeded expectations. Among the 9256 participants, $75 \%$ chose 
IUCs or implants: 46\% levonorgestrel IUC, 12\% copper-T 380A IUC and 17\% etonogestrel implant. ${ }^{4}$ There was a clinically and statistically significant reduction in abortion rates, repeat abortions and teenage birth rates.

Implants and IUCs are provided free of charge in the UK but their frequency of use is nowhere near that in the CHOICE Project. We thought that perhaps the introductory script used in the Contraceptive CHOICE Project may have played an independent role.

\section{METHODS}

We developed a simple double-sided A4 hand-out (Figures 1 and 2) with a small grant from Bayer plc. On one side was a script with pictures of copper and levonorgestrel IUCs next to a 20 pence coin and of an implant beside a hair grip (both comparisons to show actual sizes); this hand-out was developed with input from a focus group, all of whose suggestions we adopted. On the reverse side was the three-tiered effectiveness chart that had previously been published in the textbook Contraceptive Technology. ${ }^{5}$ We implemented the project in family planning (FP), abortion and antenatal clinics, as well as in general practitioner (GP) surgeries. Laminated versions of the hand-out were placed in clinical rooms. The goal was for the receptionist to give the hand-out to every woman and ask her to read it before seeing a clinician who would then ask the woman if she had read it and if she had any questions. The hand-outs were also distributed at pharmacies that delivered oral emergency contraception by patient group direction and by health visitors making home visits. We evaluated the hand-out only in FP clinics and GP practices because only for these locations are regular electronic monitoring reports generated. It was not feasible to retrieve paper records for women who had abortions or who delivered, and in fact such checking was pointless for women who delivered as no contraception was provided before discharge.
The project was initiated in FP clinics in May 2012. In GP practices the project was phased in from June to December 2012.

Fisher's exact test was used to test for differences in proportions. Calculations were performed with StatXact with Cytel Studio 8.0 ${ }^{\mathrm{TM}}$ (Cytel, Cambridge, MA, USA).

As it represented service development and quality improvement, the protocol for this intervention was exempted from research ethics committee review.

\section{RESULTS}

In GP practices there was no change in the proportion of women provided with IUCs or implants, which essentially remained flat at $2.8 \%$ over the period October 2011-November 2013. In FP clinics overall there was also no change in the proportion choosing IUCs or implants after the project initiation.

Among the Hull FP clinics only the main FP clinic, Conifer House, is open 6 days per week and has permanent sexual health staff on the reception desk. The four other satellite clinics are open for only a few hours once or twice a week. Therefore, we examined the results for Conifer House and for the other clinics separately.

At Conifer House there was an increase in the proportion of women receiving IUCs or implants of 15.0\% between October 2011-April 2012 and MayNovember 2012 [from 31.0\% (898/2895) to $35.7 \%$ (1095/3069), $p=0.0002]$, with the entire increase driven by the levonorgestrel IUC. The proportion returned to baseline in December 2012-November 2013 when there was a change in clinic procedure to reduce waiting times. Over the same time period there was a $4.6 \%$ decrease in the proportion of women choosing IUCs and implants at all satellite clinics combined [from 33.5\% (975/2910) in October 2011April 2012 to $32.0 \%$ (1025/3208) in May-November 2012, $p=0.20]$. Women obtaining a copper IUC for

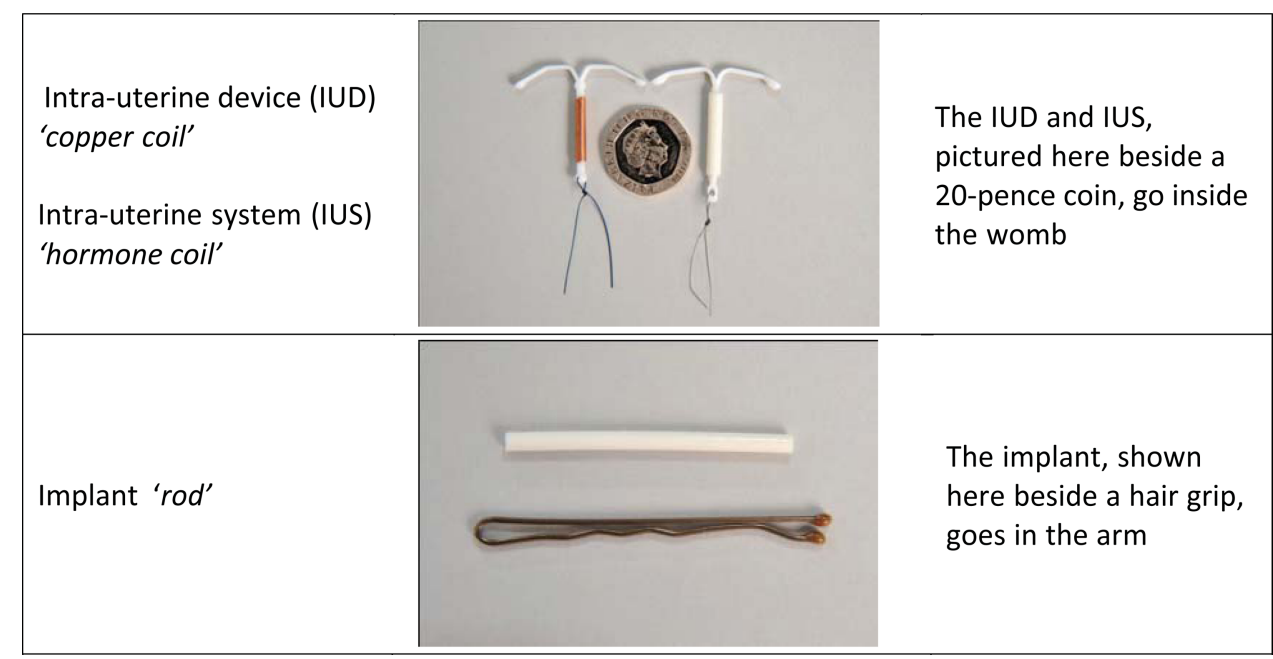

Figure 1 The long-acting reversible contraception script used for the intervention in Hull, UK. 


\section{Comparing typical effectiveness of contraceptive methods}

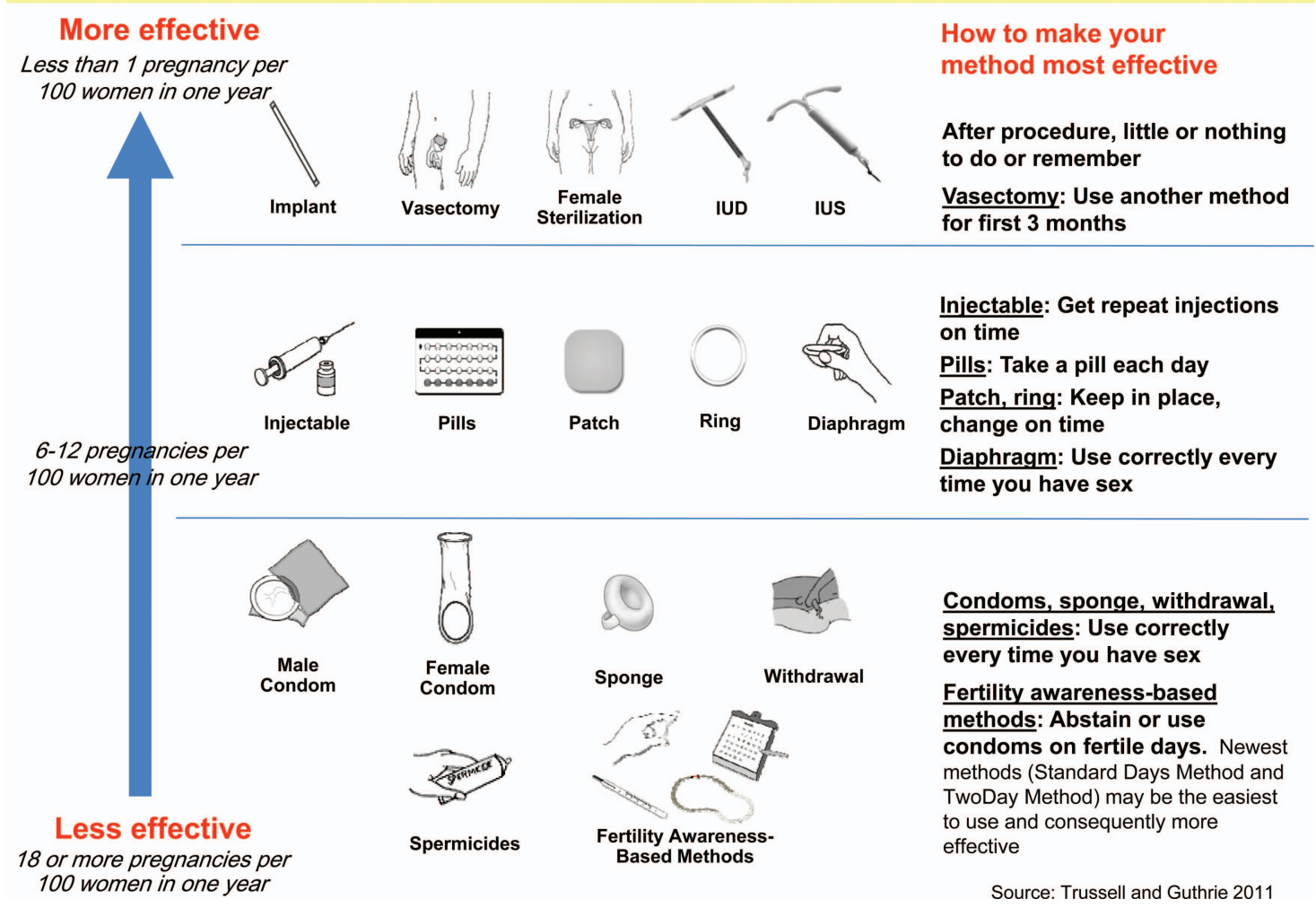

Figure 2 Three-tiered effectiveness chart for comparing the typical effectiveness of contraceptive methods reproduced from the textbook Contraceptive Technology. ${ }^{5}$ Permission to reprint this chart was obtained from Contraceptive Technology Communications and from Ardent Media.

emergency contraception would have been unlikely to be influenced by the hand-out. The results are virtually unchanged if those women are removed from the analysis.

\section{DISCUSSION}

The major limitation of our initiative was the absence of a manager to ensure that the project was actually initiated at GP clinics and satellite FP clinics, and that it was maintained long-term at Conifer House, where there was a change in clinic procedure to reduce waiting times, with the institution of an 'express' clinic run by health care assistants for 'walk-in' clients just wanting condoms, pregnancy tests and asymptomatic screening. The already overworked reception staff had the added responsibility of triaging clients and directing them to different queues, so they dropped what they considered to be their least important task, namely dispensing hand-outs. We did not discover this until we talked with the reception staff after seeing our results. Ongoing staff engagement would probably have been enhanced if we had fed back the positive results as they were observed. In the four satellite clinics not all reception staff are dedicated purely to sexual health, and staff engagement for project work in general is more challenging. Again, it was not until we saw our results that we discovered that the initiative had been poorly implemented. Likewise, we believe that the initiative was poorly implemented in GP practices, perhaps because the Conifer House logo appeared on the hand-out and the GPs therefore felt threatened by competition.

The impact of such an initiative would possibly be greater where baseline use of IUCs and implants is lower than in Hull. The St Louis CHOICE Project offered same-day placement of IUCs and implants that undoubtedly increased uptake; same-day placement of IUCs (bar the placement of emergency IUCs) is not routinely available in Hull. The CHOICE Project also offered far more time-intensive counselling than we are able to provide in Hull.

\section{CONCLUSIONS}

We have shown that a very simple and cheap intervention can increase uptake of IUCs and implants; this must be by far the most cost-effective long-acting reversible contraception intervention on record. The variation in uptake with the 'off-on-off' implementation considerably strengthens our conclusion that the increase we observed was due to this simple intervention. However, lessons learned were that a supervisor/ manager is needed for planning, monitoring and providing prompt feedback; that a dedicated team (reception staff and clinicians) is needed for 
implementation; and that ongoing engagement of the reception staff and clinicians is essential for successful continuation of such an initiative.

Funding This work was supported by an National Institute of Child Health and Human Development/National Institutes of Health (NICHD/NIH) grant for Infrastructure for Population Research at Princeton University, Grant R24HD047879 (James Trussell) and by a small grant from Bayer plc.

Competing interests None.

Provenance and peer review Not commissioned; externally peer reviewed.

\section{REFERENCES}

1 National Institute for Health and Clinical Excellence. Long-acting Reversible Contraception: The Effective and
Appropriate Use of Long-acting Reversible Contraception (Clinical Guideline 30). October 2005. http://www.nice.org.uk/ CG030 [accessed 4 March 2014].

2 Trussell J, Wynn LL. Reducing unintended pregnancy in the United States. Contraception 2008;77:1-5.

3 Secura GM, Allsworth JE, Madden T, et al. The Contraceptive CHOICE Project: reducing barriers to long-acting reversible contraception. Am J Obstet Gynecol 2010;203:115.e1-115.e7.

4 Peipert JF, Madden T, Allsworth JE, et al. Preventing unintended pregnancies by providing no-cost contraception. Obstet Gynecol 2012;120:1291-1297.

5 Trussell J, Guthrie KA. Choosing a contraceptive: efficacy, safety, and personal considerations. In: Hatcher RA, Trussell J, Nelson AL, et al. (eds), Contraceptive Technology (Twentieth Revised Edition). New York, NY: Ardent Media, 2011:45-74. 